\title{
Study of 5G Technology Support in Smart Vehicle System
}

\author{
D. Karunkuzhali ${ }^{1}$, P. Saravanan ${ }^{2}$, R. Ohmsakthi Vel ${ }^{3}$, B. Muthuvel ${ }^{4}$, K. Latha ${ }^{5}$ \\ \{karunkuzhali@gmail.com¹, saravanan.ece@sairamit.edu.in², ohmohms@gmail.com³ \\ muthuvel.bmv7@yahoo.co.in ${ }^{4}$,klathasn@gmail.com $\left.{ }^{5}\right\}$

\begin{abstract}
${ }^{1}$ Professor, Department of Information Technology, Panimalar Engineering College, Chennai, India, Technology, Chennai, India, ${ }^{3}$ Assistant Professor, Department of Mechatronics Engineering, Agni College of Technology, Chennai, India, ${ }^{4}$ Professor, Department of Electrical and Electronics

${ }^{5}$ Assistant Professor, Department of Computer Science and Engineering, Sri Sairam Engineering
\end{abstract} \\ ${ }^{2}$ Associate Professor, Department of Electronics and Communication Engineering, Sri Sairam Institute of \\ Engineering, Bonam Venkata Chalamayya Institute of Technology and Science, Amalapuram, India, \\ College, Chennai, India
}

\begin{abstract}
The projected exploration study uncovers the significant jobs of $5 \mathrm{G}$ advances for the keen vehicle framework. 5G innovations will empower transport vehicles to associate with the organize and speak with one another, guaranteeing super high unwavering quality and low idleness. Empowering this sort of network will profit by a troublesome new application that will permit to improve driving proficiency and accelerate street security. The primary fundamental aftereffects of the information demonstrate the transformation of $5 \mathrm{G}$ innovations to the savvy vehicle framework in various nations. Additionally, a business viewpoint is given, where the change of the car area because of $5 \mathrm{G}$ is talked about. $5 \mathrm{G}$ will give the essential framework to establish a savvy web of vehicle climate, which will push the activity and limit necessities of the vehicle organization to their limits. The momentum inexact exploration study incorporates different calculations for $\mathrm{V} 2 \mathrm{X}$ and the Nivebase multinomial calculation is about $82.72 \%$. The estimating research paper additionally recorded $5 \mathrm{G}$ innovative executions in various nations.
\end{abstract}

Keywords: Algorithm, 5G Technol, G, V2X, Simulation, Chipsets.

\section{Introduction}

Self-governing and trend setting innovation in vehicles is as of now changing the auto business. The effect of future vehicle advances has been seriously anticipated and development is sprouting in research-related spaces. Expanding street wellbeing, smoothed out movement time, altered offices, energy the board and stopping lessen social advantages. A significant benefit of auto tommation of Om tomobiles is the improvement in security, which normally comes when we eliminate the foundation of most traffic occurrences: human conduct out and about. Meeting wellbeing necessities and settling on choices for multi-f optim streamlined driving is upheld by sensors that are inside the vehicle or that live in the climate (counting different vehicles). Moreover, data is traded with the vehicle's computerized twin, as it might contain extra data identified with traffic street $\mathrm{C}$, street work, climate and different conditions. 
Correspondence between the vehicle and any remaining outside bodies is upheld by remote correspondence advances, one of the main of which is $5 \mathrm{G}$, which ensures administration at the last stage.

\section{Related work}

Propaganda channel is a major performance factor that affects any communication system. The fast speed of the vehicles, the dynamic surroundings always get messed upFixed and mobile scatter, and low antenna ights height V2X poses challenges for communications that are unique compared to other communication systems. Moreover, the various 2 of the application have the notion that the 5G V2X system is intendedSupport - From basic safety applications [1 - 1-3] to high precision radio positioning, to advanced cooperative automated driving applications (e.g., platooning, cooperative intersection control, etc.) - results in significantly different requirements.

\section{In terms of channel modeling.}

Most of the leading examples of GBD models Dello are based on ray tracing / ray launching, while GP G.P. The model adopted by [-6--6], which is based on the development of the WINNER framework, is the most widely used GBD model. However, so far, []] in 3 G.P.P. Some of the models do not implementConsidering the effect of dual mobility on key V2X features, such as fast fading parameters (required for V2V), they consider V2X-specific synergies (highway, street-level urban, road unit-to-vehicle, V2V). Don't take, and don't. Considered V2X-Spacey-C antenna.

\section{Research Methods}

This section presents the content and methods of this inferential research work. Dataset for 5G technology implementation $\mathrm{G}$ implementation is taken from Statistia.com. In this study, various algorithms and networks have been used for the analysis of 5G technology in smart vehicle systems.

The Weka 3.8.9 has implemented to get the optimal solution of the above dataset. The below approaches have implemented and got optimal solution.

- BayesNet:Bayes

- NaiveBayes:

- NaiveBayesMultinomial:

- NaiveBayesMultinomialText:

- NaiveBayesMultinomialUpdateable:

Integrated Moving Networks 
The purpose of mobile base stations is to effectively provide low latency services to vehicle users, data rates and modern, well-insulated vehicles with low loss of carrier frequency (in $25 \mathrm{~dB}$ ). Millimeter-waves (G $100 \mathrm{GHz}$ )

Table 1. Basic details of $5 \mathrm{G}$ technology in smart vehicle system

\begin{tabular}{|l|l|l|l|}
\hline Locality & Rural & Urban & Highway \\
\hline Link Type (V2X) & V2P & V2P & V2P \\
& V2V & V2V & V2V \\
& V2I & V2I & V2I \\
\hline
\end{tabular}

\section{Results And Discussion}

The analysis results indicate the active and inactive $5 \mathrm{~g}$ technology implementation in various countries. Technology adopted countries is about 61 and 131 countries, active and inactive respectively

Figure 1. Status of $5 \mathrm{G}$ Technology

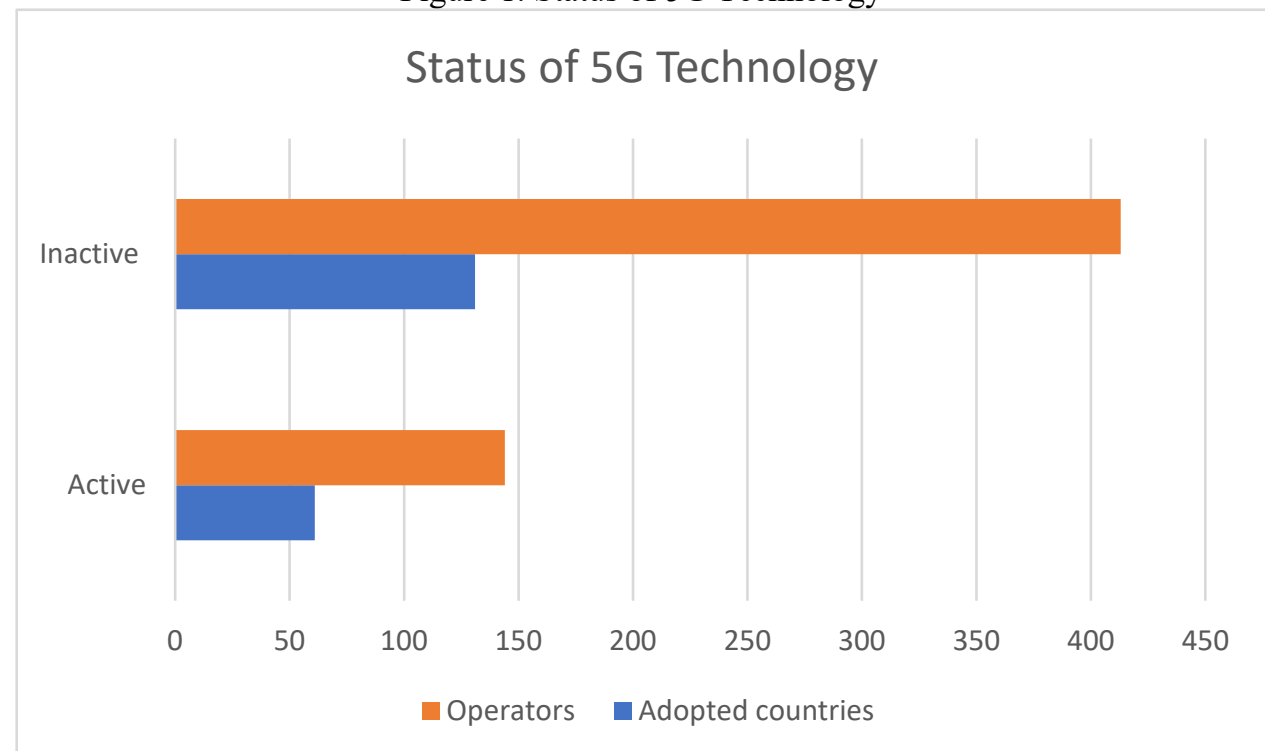

Advanced V2X channel models have been developed that cover a wide range of scenarios and fields based on data analysis and analysis. [Species -] Two recent studies of this species are available. Given the number of cases, locations and classification of models w.r.t. Modeling methods (Figures 1 and 2), can be thousandsCombination, which makes it difficult to make the right choice of model parameters. The use of channel models with wireless system design materials to create sensitivities orSimulating end-to-end system performance when chipsets rating, collecting performance statistics, and evaluating protocol applications.

A detailed, informative recipeTo enable programmers to select the appropriate $\mathrm{V} 2 \mathrm{X}$ channel model is not explicitly available in textbooks. I gave you such a recipe, this section summarizes 
The main components for selecting the right channel models dales, which are the beginning of a more detailed section, distance analysis and additional measurements and modeling that you will5GCAR is built within the project.

Table 2. Algorithm used in smart vehicle system

\begin{tabular}{|l|l|l|l|}
\hline Algorithm & V2P & V2V & V2I \\
\hline BayesNet & $79.14 \%$ & $81.14 \%$ & $82.72 \%$ \\
\hline NaiveBayes & $79.23 \%$ & $78.14 \%$ & $79.94 \%$ \\
\hline NaiveBayesMultinomial & $79.94 \%$ & $82.72 \%$ & $79.57 \%$ \\
\hline NaiveBayesMultinomialText & $79.57 \%$ & $79.94 \%$ & $79.57 \%$ \\
\hline NaiveBayesMultinomialUpdateable & $74.67 \%$ & $79.57 \%$ & $74.67 \%$ \\
\hline
\end{tabular}

Figure 2. Algorithm used in smart vehicle system

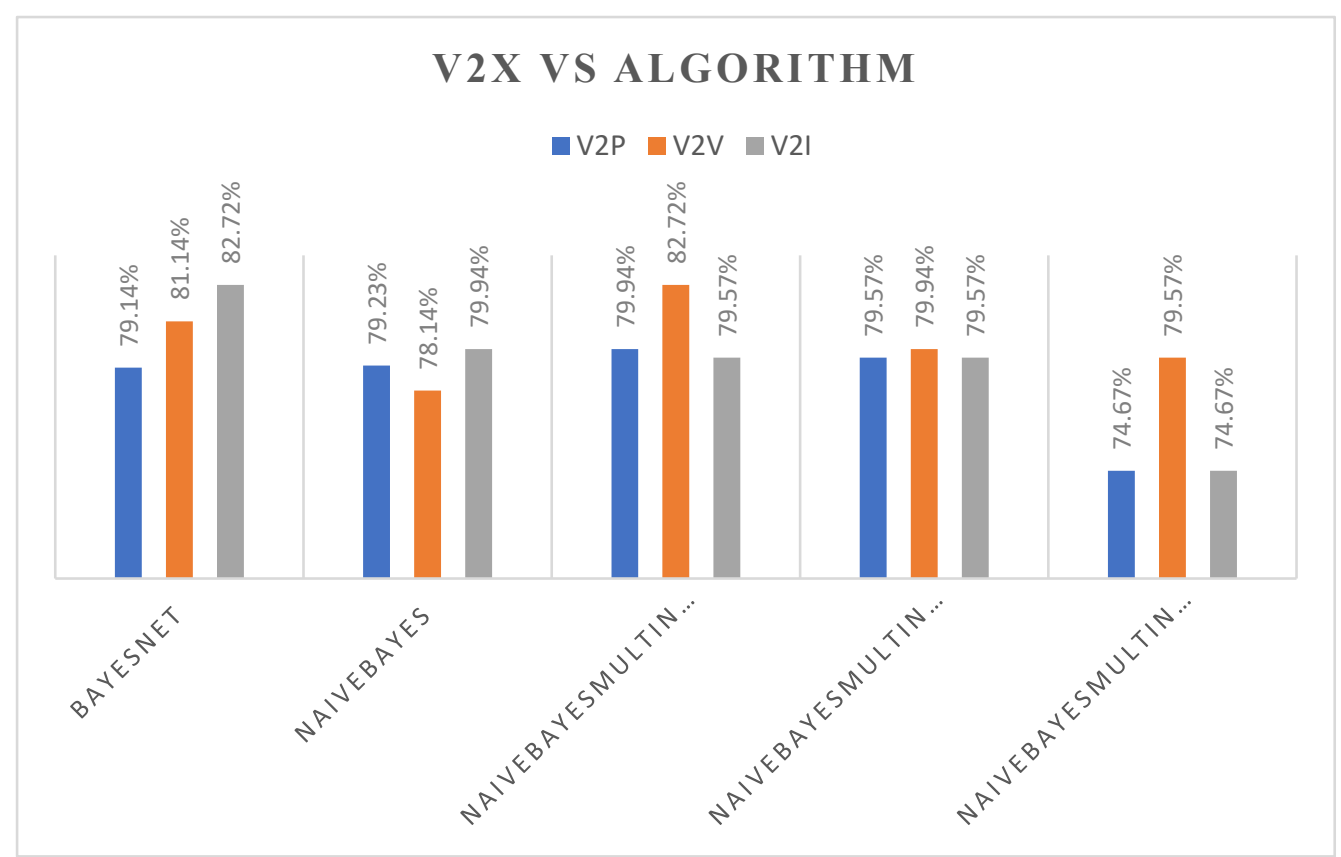

The table 2 above clearly shows the correct call rates for various methods namely BayesNet, NaiveBayes, NaiveBayes Multinomial, NaiveBayesMultinomialText, NaiveBayes Multinomial Updateable and NaiveBayesUpdateabl. All of these algorithms belong to the Bayes program. The diagram above represents that BayesNet produces V2P - 79.14\%, V2V81.14\%, V2I - 82.74\%, NaiveBayes owns V2P - 79.24\%, V2V- 78.14\%, V2I - 79.94\%, NaiveBayesMultinomial 7 -14, V2V - 81.14\%, V2I - 82.74\%, NaivBayesMultinomialText gives V2P value - 79.94\%, V2V- 82.72\%, V2I - 79.57\%, NaiveBayesMultinomialUpdateable gives V2P - 79.59\%, V2P - 79.57\% . All leading Bayes categories have produced an accuracy rate of more than $74 \%$ and the highest accuracy rate is $82.72 \%$ generated by the NaivebayesMultinomial algorithm.

A key feature of $5 \mathrm{G}$ in smart automotive system, user data rate, Latency, Coverage, Capacity, cost of data types and sensor devices used, of which GNSS is the Global Navigation 
Satellite System, and IMU is Inertial Measurement Unit. The shows themselves were successful with $5 \mathrm{G}$-based communication working effectively on a live network. Self-driving vehicles interact with its Digital Twin and successfully perform scheduled SCiL tests, e.g., fastening the brakes in front of visible and real pedestrians, tracking, and avoiding real and real vehicles [8-10].

The expansion of this exhibition took place on the M86 highway, where cars run at different speeds and perform different functions - and every stop related to RSU details was recorded. To support future SciL experiments, this sensor data can also be played to insert the Digital Twin into the visual space of the M86 [11 - 12].

To test the current power of $5 \mathrm{G}$ in real-life situations, we have developed a series of $5 \mathrm{G}$ NSA 3.X architecture options, targeting the Round-Trip-Time distribution and the InterArrival Time package. This helped to identify the latency capacity of the current 5G NSA network. We found that a variety of V2X scenarios - from planning to integration data integration and much more - are already out of support based on standard requirements. Advantages of $5 \mathrm{G}$ connectivity, in the new concept of Automotive Internet during low latency, extremely high bandwidth and reliability [13 - 15].

\section{Conclusion}

Smart technology has important points in achieving a 5G compatible car. The main theme is the attributes of the basic V2X channels. In addition, we have introduced the building squares for the V2X cell layout. Flexible organizational engineering has been introduced all together to help the ongoing management of V2X. We have further investigated the potential for use of vehicles as portable power stations as part of that flexible engineering. The fog detection was introduced in relation to the corresponding vehicle, and finally, a biological system that included a compatible vehicle was investigated. Using $5 \mathrm{G}$ technology can reduce delays that will improve access to smart car technology. We assess and highlight the problems and shortcomings of current mobile technologies in relation to these operating conditions and identify how 5G will overcome those shortcomings. We also identified open research problems and provided future indicators to deal with those problems.

\section{References}

[1] Feddes, G.; Kuipers, J. Software Driving License; Intertraffic: Amsterdam, The Netherlands, 2018.

[2] ZalaZONE. Where Innovation Leads. Available online: https://zalazone.hu/en/ (accessed on 18 December 2020)

[3] Szalay, Z.; Hamar, Z.; Nyerges, A. Novel design concept for an automotive proving ground supporting multilevel CAV development. Int. J. Veh. Des. 2019, 80, 1-22.

[4] Horváth, M.; Lu, Q.; Tettamanti, T.; Török, A.; Szalay, Z. Vehicle-In-The-Loop (VIL) and Scenario-In-The-Loop (SCIL) Automotive Simulation Concepts from the Perspectives of Traffic Simulation and Traffic Control. Transp. Telecommun. J. 2020, 20, 153-161.

[5] Tihanyi, V.; Szalay, Z. Autonomous vehicle platform for demonstration purposes. In Proceedings of the Advanced Manufacturing and Repairing Technologies in Vehicle Industry, Visegrad, Hungary, 17-19 May 2017; pp. 144-148.

[6] Kozma, D.; Soos, G.; Ficzere, D.; Varga, P. Communication Challenges and Solutions between Heterogeneous Industrial IoT Systems. In Proceedings of the 2019 15th International 
Conference on Network and Service Management (CNSM), Halifax, NS, Canada, 21-25 October 2019.

[7] Németh, H.; Háry, A.; Szalay, Z.; Tihanyi, V.; Tóth, B. Proving Ground Test Scenarios in Mixed Virtual and Real Environment for Highly Automated Driving. In Mobilität in Zeiten der Veränderung: Technische und BetriebswirtschaftlicheAspekte; Proff, H., Ed.; Springer: Wiesbaden, Germany, 2019; pp. 199-210.

[8] Heineke, K.; Ménard, A.; Södergren, F.; Wrulich, M. Development in the Mobility Technology Ecosystem-How Can 5G Help? McKinsey and Company. 2019. Available online: https://www.mckinsey.com/industries/automotive-and-assembly/ourinsights/development-inthe-lmobility-technology-ecosystem-how-can-5g-help (accessed on 18 December 2020).

[9] Ge, X. Ultra-Reliable Low-Latency Communications in Autonomous Vehicular Networks. IEEE Trans. Veh. Technol. 2019, 68, 5005-5016.

[10] Varga, P.; Peto, J.; Frankó, A.; Balla, D.; Haja, D.; Janky, F.; Soos, G.; Ficzere, D.; Maliosz, M.; Toka, L. 5G support for Industrial IoT Applications-Challenges, Solutions, and Predicted research gaps. Sensors 2020, 20, 828.

[11] Soós, G.; Ficzere, D.; Varga, P.; Szalay, Z. Practical 5G KPI Measurement Results on a NonStandalone Architecture. In Proceedings of the NOMS 2020-2020 IEEE/IFIP Network Operations and Management Symposium, Budapest, Hungary, 20-24 April 2020; pp. 1-5.

[12] 3GPP. Service Requirements for the 5G System; TS 22.261 v17.0.1; 3rd Generation Partnership Project: Valbonne, France, 2019.

[13] 3GPP. Service Requirements for Cyber-Physical Control Applications in Vertical Domains; TS 22.104 v17.2.0; 3rd Generation Partnership Project: Valbonne, France, 2019.

[14] 3GPP. Service Requirements for Enhanced V2X Scenarios; TS 22.186 v16.2.0; 3rd Generation Partnership Project: Valbonne, France, 2019.

[15] 3GPP. Mobile Communication System for Railways; TS 22.289 v16.1.0; 3rd Generation Partnership Project: Valbonne, France, 2019. 